\title{
TOPOLOGICAL PROPERTIES OF SPACES ORDERED BY PREFERENCES
}

\author{
J. C. R. ALCANTUD
}

(Received 24 April 1997 and in revised form 22 November 1997)

\begin{abstract}
In this paper, we analyze the main topological properties of a relevant class of topologies associated with spaces ordered by preferences (asymmetric, negatively transitive binary relations). This class consists of certain continuous topologies which include the order topology. The concept of saturated identification is introduced in order to provide a natural proof of the fact that all these spaces possess topological properties analogous to those of linearly ordered topological spaces, inter alia monotone and hereditary normality, and complete regularity.
\end{abstract}

Keywords and phrases. Saturated identifications, order topology, GPO-spaces, POTS, monotonically normal, normal, regular.

1991 Mathematics Subject Classification. 06F30, 54F05.

1. Introduction. This paper is devoted to perform a thorough study of the topological properties of spaces ordered by preferences (asymmetric, negatively transitive binary relations). The general problem of studying the topologies associated with ordered spaces, among which the most popular are the order and the interval topologies, has proved to be rather difficult and, thus, the literature abounds with results on the behavior of such topologies (cf. Erné [6], Kolibiar [12], Nachbin [17], Northam [18]). However, the particular case of linear orders has been deeply analyzed and it has been shown that they possess a rich topological structure. We prove that spaces ordered by preferences display most of the topological properties of linearly ordered spaces. Besides, in our study, we also consider separability and the axioms of countability.

Let us recall the main results in this line which concern linear orders. Any linearly ordered topological space (abbreviated: LOTS) is $T_{1}$ and hereditarily normal. See, e.g., Steen [13]. This class of topological spaces or, in general, those whose topology is related in some sense to a linear order, have been widely investigated (cf. Birkhöff [3], Duda [4], Eilenberg [5], Frink [19], Lynn [15]). A particularly interesting case is that of GO-spaces (which stands for Generalized Ordered spaces) because they include LOTS and satisfy properties analogous to theirs. In fact, a remarkable result by R. W. Heath and D. J. Lutzer in [10] shows that every GO-space is $T_{1}$ and monotonically normal, which is an improvement of the aforementioned result that LOTS are hereditarily normal. On the other hand, van Dalen and Wattel [20] characterized those topological spaces that are LOTS or GO-spaces. The monographies of Faber [7] and van Wouwe [21] are devoted to the study of the topological properties of GO-spaces, and especially to metrizability.

In this paper, we define and analyze the natural generalization of the concepts of 
LOTS and GO-space to the preference case, namely, POTS (for Preference-Ordered Topological Space) and GPO-space (for Generalized Preference-Ordered space), respectively.

For POTS, some results have been obtained, e.g., by Mehta, who provided characterizations of separability and second countability in [16], and by Herden, who gave a characterization of second countability in [11]. Here, we show that GPO-spaces, which are not $T_{0}$ unless the order is linear, are (hereditarily) normal and (completely) regular. Furthermore, they satisfy a condition that is equivalent to monotone normality for $T_{1}$-spaces. The procedure for obtaining these results consists of studying the properties of certain maps that we call saturated identifications. The projection of a GPO-space onto a suitable GO-space is a saturated identification. As well as providing the aforementioned facts, this enables us to characterize many topological properties of GPO-spaces via the GO-space case. Other properties that had not been studied in GO-spaces, namely, topological separability and the first and second axioms of countability, are characterized for GPO-spaces in Alcantud [1].

This paper is organized as follows. We introduce saturated identifications, study their properties, and recall some topological definitions in Section 2. After fixing the terminology on partially ordered sets, we define GPO-spaces as a natural generalization of GO-spaces and we give two characterizations of this concept in Section 3. We also link saturated identifications and the subject of our study. Those results are used to analyze GPO-spaces and POTS in Section 4.

2. General properties of saturated identifications. Unless otherwise specified, in this section, we follow the terminology of Wilansky [22].

DEFINITION 2.1. Let $(X, \tau)$ and $\left(X^{\prime}, \tau\right)$ be topological spaces. We say that a quotient map $f:(X, \tau) \longrightarrow\left(X^{\prime}, \tau^{\prime}\right)$ is a saturated identification if, for each $A \in \tau, A=f^{-1} f(A)$. We also say that $f: X \longrightarrow X^{\prime}$ is a saturated identification with the topologies $\tau, \tau^{\prime}$.

Every saturated identification $f:(X, \tau) \longrightarrow\left(X^{\prime}, \tau^{\prime}\right)$ is both open and closed. Furthermore, whenever $C$ is a closed subset of $X$, we have $C=f^{-1} f(C)$.

Because an open (closed) continuous onto map is a quotient map, saturated identification can be redefined as an open (closed) continuous onto map with $A=f^{-1} f(A)$ for all $A \in \tau\left(C=f^{-1} f(C)\right.$ for all $C$ closed $)$.

DEFINITION 2.2. If $(X, \tau)$ is a topological space, an equivalence relation $R$ on $X$ saturates $\tau$ if $x \in A \in \tau$ and $y R x$ implies $y \in A$.

If $f$ is the quotient map associated with an equivalence relation $R$ on $X$, the condition that $A=f^{-1} f(A)$ for all $A \in \tau$ is equivalent to $R$ saturates $\tau$.

LEMMA 2.1. If $f:(X, \tau) \longrightarrow\left(X^{\prime}, \tau^{\prime}\right)$ is a saturated identification and $\tau$ is $T_{0}$, then $f$ is a homeomorphism.

Proof. The map $f$ must be injective: if there exists $x \neq y$ with $f(x)=f(y)$, then, for each $x \in A \in \tau, y \in f^{-1} f(A)=A$, and, similarly, for each $y \in A^{\prime} \in \tau$, we have $x \in A^{\prime}$, contradicting the fact that $\tau$ is $T_{0}$. This ends the proof.

DEFINITION 2.3. A topological space $(X, \tau)$ is monotonically normal $(\mathrm{MN})$ if there 
exists a monotone normality operator $D$, assigning to each ordered pair $(H, K)$ of closed disjoint sets an open set $D(H, K)$, and satisfying:

(MN.1) for all $(H, K)$ ordered pair of closed disjoint sets: $H \subseteq D(H, K) \subseteq \operatorname{cl}(D(H, K))$ $\subseteq X \backslash K$.

(MN.2) for all $(H, K),\left(H^{\prime}, K^{\prime}\right)$ ordered pairs of closed disjoint sets with $H \subseteq H^{\prime}$, $K^{\prime} \subseteq K: D(H, K) \subseteq\left(H^{\prime}, K^{\prime}\right)$.

Without loss of generality, we may further assume that $D$ satisfies:

(MN.3) for all $(H, K)$ ordered pair of closed disjoint sets: $D(H, K) \cap D(K, H)=\varnothing$ (cf. Heath et al. [10, Def. 2.1 and Lem. 2.2]).

A closely related property (as defined in Gruenhage [9]) is the following:

DEFINITION 2.4. A topological space satisfies property $\mathrm{MN}^{\prime}$ if there exists an operator $M$ which assigns to each $(x, U)$ ordered pair with $x \in U$ open, an open set $M(x, U)$ containing $x$, in such a way that $M(x, U) \cap M(y, V) \neq \varnothing$ implies $x \in V$ or $y \in U$. Without loss of generality, we can assume that, for all $U \subseteq V$ open containing $x, M(x, U) \subseteq M(x, V)$.

In general, $\mathrm{MN}^{\prime}$ implies $\mathrm{MN}$. Moreover, $\mathrm{MN}$ and $\mathrm{MN}^{\prime}$ are equivalent in $T_{1}$ spaces (cf. Gruenhage [9, Thm. 5.19]).

Recall the well-known characterization (cf. Willard [23] 15B) that a topological space is hereditarily normal if and only if every two separated subsets of it admit disjoint neighborhoods (i.e., for any $A$ and $B$ subsets, if $\operatorname{cl}(A) \cap B=\varnothing=A \cap \operatorname{cl}(B)$, then there exists $A \subseteq U, B \subseteq V, U$ and $V$ open, such that $U \cap V=\varnothing$ ).

It is known that (countable) compactness, Lindelöff, separability, connectedness are preserved by continuous onto maps; local connectedness is preserved by quotient maps; first and second countability and local compactness are preserved by open and continuous onto maps; (hereditary, monotone) normality are preserved by closed and continuous onto maps; regularity is preserved by open and closed continuous onto maps, and perfect onto maps. Therefore, all these properties are preserved by saturated identifications.

In addition, saturated identifications transfer properties from the quotient topol$\operatorname{ogy} \tau^{\prime}$ to the original topology $\tau$ too. This is proved in Theorem 2.1. Some of those properties are a consequence of the following fact, whose proof is immediate:

LEMMA 2.2. If $f:(X, \tau) \longrightarrow\left(X^{\prime}, \tau^{\prime}\right)$ is a saturated identification, then $\tau$ is the weak (or initial) topology by $f$ for $X$.

Therefore, if $f$ is a saturated identification and $\tau^{\prime}$ is normal, completely regular or regular, then $\tau$ is the same (cf. Wilansky [22]).

THEOREM 2.1. Let $f:(X, \tau) \longrightarrow\left(X^{\prime}, \tau^{\prime}\right)$ be a saturated identification. Then

(i) $T$ is first (second) countable if and only if $\tau^{\prime}$ is first (second) countable.

(ii) $T$ is separable if and only if $\tau^{\prime}$ is separable.

(iii) $\tau$ is compact (countably compact, Lindelöff, locally compact) if and only if $\tau^{\prime}$ is compact (countably compact, Lindelöff, locally compact).

(iv) $\tau$ is (locally) connected if and only if $\tau^{\prime}$ is (locally) connected. Actually: $C \subseteq X^{\prime}$ is connected if and only if $f^{-1}(C)$ is connected, $C \subseteq X$ is connected if and only if $f(C)$ is connected. 
(v) $\tau$ is (hereditarily, monotonically) normal if and only if $\tau^{\prime}$ is hereditarily, monotonically) normal.

(vi) $\tau$ is regular if and only if $\tau^{\prime}$ is regular.

(vii) if $\tau^{\prime}$ is completely regular, then $\tau$ is completely regular.

Proof. The sufficiency of the conditions in (i) to (v), as well as (vi) and (vii), have already been justified. Now, we proceed to prove the necessity in conditions (iv) and (v), which are used later. Proofs for the remaining implications can be found in Alcantud and Gutiérrez [2].

(iv) Suppose that $X^{\prime}$ is locally connected; we will show that $X$ is locally connected. Given $x \in X$, we must find a base of connected neighborhoods of $x$. If $\left\{V_{\alpha}\right\}_{\alpha \in \Lambda}$ is a base of connected neighborhoods of $f(x)$ it has been shown in the proof of (i) that $\left\{f^{-1}\left(V_{\alpha}\right)\right\}_{\alpha \in \Lambda}$ is a base of neighborhoods of $x$. It only remains to see that it consists of connected sets.

To check this, firstly, we prove that if $C \subseteq X^{\prime}$ is connected, $f^{-1}(C)$ is connected. Otherwise, there would be disjoint nonvoid sets $A, B \subseteq X$, open in the relative topology of $f^{-1}(C)$, such that $f^{-1}(C)=A \cup B$. Therefore, there exist $A^{\prime}, B^{\prime} \in \tau$ such that $A=$ $A^{\prime} \cap f^{-1}(C)$ and $B=B^{\prime} \cap f^{-1}(C)$. One may check that $f\left(A^{\prime} \cap f^{-1}(C)\right)=f\left(A^{\prime}\right) \cap C$ and, thus, this set is open in the relative topology of $C$ (and the same is true for $B$ ). The sets $f\left(A^{\prime} \cap f^{-1}(C)\right)$ and $f\left(B^{\prime} \cap f^{-1}(C)\right)$ disconnect $C$ since

$$
C=f f^{-1}(C)=f(A) \cup f(B)=f\left(A^{\prime} \cap f^{-1}(C)\right) \cup f\left(B^{\prime} \cap f^{-1}(C)\right)
$$

and they are disjoint (otherwise, there exists $y \in f\left(A^{\prime} \cap f^{-1}(C)\right) \cap f\left(B^{\prime} \cap f^{-1}(C)\right)$, and so $y=f\left(a^{\prime}\right)=f\left(b^{\prime}\right)$ for some $a^{\prime} \in A^{\prime} \cap f^{-1}(C), b^{\prime} \in B^{\prime} \cap f^{-1}(C)$. From $a^{\prime} \in f^{-1} f(b)$, follows the contradiction $\left.a^{\prime} \in A^{\prime} \cap B^{\prime} \cap f^{-1}(C)=A \cap B=\varnothing\right)$.

In particular, we have obtained that $X$ is connected if $X^{\prime}$ is connected, and that $C \subseteq X^{\prime}$ is connected if and only if $f^{-1}(C)$ is connected (the necessary condition holds because $f$ is onto and continuous).

It remains to see that for all $C \subseteq X, f(C)$ connected implies $C$ connected. So far, we know that $f(C)$ connected implies $D_{C}:=f^{-1} f(C)$ connected. The proof is completed if we show that $C$ disconnected implies $D_{C}$ disconnected. For this, assume that there exist $A, B \in \tau$ such that $C=(A \cap C) \cup(B \cap C)$ with $A \cap B \cap C=\varnothing, A \cap C \neq \varnothing \neq B \cap C$. The sets $A \cap D_{C}$ and $B \cap D_{C}$ disconnect $D_{C}$ since they partition $D_{C}$ (if $x \in D_{C}=f^{-1} f((A \cap$ $C) \cup(B \cap C)$ ), then either $x \in f^{-1} f(A)=A$ or $x \in f^{-1} f(B)=B$ ) and they are disjoint (if there exists $x$ in $D_{C} \cap A \cap B$, there exists an element $c \in C$ such that $f(x)=f(c)$, and then $x \in A, x \in B$ imply $c \in f^{-1} f(x) \subseteq f^{-1} f(A)=A$ and, analogously, $c \in B$ holds from $x \in B$. This contradicts the fact that $A \cap B \cap C=\varnothing)$.

(v) Both implications have been justified for normality.

We now verify the necessary condition for hereditary normality using the aforementioned characterization. Suppose that $\tau^{\prime}$ is hereditarily normal. Let $A, B$ be separated subsets of $X$; then $f(A)$ and $f(B)$ are separated (by symmetry, we only need to show that: $\operatorname{cl}(f(A)) \cap f(B)=\varnothing): f$ closed and continuous implies $f(\operatorname{cl}(A))=\operatorname{cl}(f(A))$ and, therefore, $\operatorname{cl}(f(A)) \cap f(B)=f(\operatorname{cl}(A)) \cap f(B)=\varnothing$ (if there exists $x^{\prime} \in f(\operatorname{cl}(A)) \cap$ $f(B)$, then $x^{\prime}=f(b)=f(a)$ for some $b \in B$ and $a \in \operatorname{cl}(A)$. Thus, $b \in f^{-1}\left(x^{\prime}\right) \subseteq$ 
$f^{-1}(f(\operatorname{cl}(A))=\operatorname{cl}(A)$, which contradicts $\operatorname{cl}(A) \cap B=\varnothing)$. Therefore, there exist disjoint $U^{\prime}, V^{\prime} \in \tau$ such that $f(A) \subseteq U^{\prime}, f(B) \subseteq V^{\prime}$. Now, $A \subseteq f^{-1}(f(A)) \subseteq f^{-1}\left(U^{\prime}\right) \in$ $\tau, B \subseteq f^{-1}\left(V^{\prime}\right) \in \tau$ and $f^{-1}\left(U^{\prime}\right) \cap f^{-1}\left(V^{\prime}\right)=\varnothing$.

We now verify the necessary condition for monotone normality. Suppose that $\tau^{\prime}$ is monotonically normal. Take $D^{\prime}$ a monotone normality operator on $X^{\prime}$. For any $H$ and $K$ disjoint closed subsets of $X$, let $D(H, K)=f^{-1}\left(D^{\prime}(f(H), f(K))\right)$ (notice that $f(H)$ and $f(K)$ are closed and disjoint too). The operator $D$ so defined clearly satisfies (MN.2). Let us see that $D$ also satisfies (MN.1). Because $H=f^{-1} f(H)$ and $f(H) \subseteq$ $D^{\prime}(f(H), f(K))$, it follows that $H \subseteq f^{-1}\left(D^{\prime}(f(H), f(K))\right)=D(H, K)$. Furthermore, $D(H, K)=f^{-1}\left(D^{\prime}(f(H), f(K))\right)$ implies that $\operatorname{cl}(D(H, K)) \subseteq f^{-1}\left(\operatorname{cl}\left(D^{\prime}(f(H), f(K))\right)\right)$ by the continuity of $f$ and, therefore, $\operatorname{cl}(D(H, K)) \subseteq f^{-1}\left(X^{\prime} \backslash f(K)\right)=X \backslash K$ since $\operatorname{cl}\left(D^{\prime}(f(H), f(K))\right) \subseteq X^{\prime} \backslash f(K)$.

3. GPO-spaces: definitions and first properties. Recall some definitions. A binary relation $<$ on a set $X$ is asymmetric if, for any $x, y \in X, x<y$ implies that $y<x$ is false. We say that it is negatively transitive if, for each $x, y, z \in X$ such that $x<y$ is false and $y<z$ is false, then $x<z$ is false. An asymmetric, negatively transitive binary relation $<$ on a set is a preference. If a preference $<$ satisfies that either $x<y$ or $y<x$ for any distinct elements $x$ and $y$, then $<$ is called a linear (or total) order. We say that $X$ is a set ordered by a preference $<$ and $X$ is linearly ordered by $<$, respectively. We may associate with any preference $<$ an equivalence relation called indifference relation. It is denoted by $\sim$ and defined by $x \sim y$ if and only if not, $x<y$, not $y<x$. By $x \leq y$, we mean $x<y$ or $x \sim y$.

In what follows, if a preference is denoted by $<$, then $\sim$ stands for the indifference that $<$ induces. If the preference is denoted by $<^{\prime}$, the symbol $\sim^{\prime}$ is used instead.

Every preference $<$ on a set $X$ satisfies (cf. Fishburn [8, Thm. 2.1]):

(P.1) for every $a, x, y, z \in X: a \sim y<z$ implies $a<z$ and $x<y \sim a$ implies $x<a$,

(P.2) for every $x, y \in X$ : exactly one of $x<y, x \sim y$ or $y<x$ is true.

A transitive, asymmetric (or irreflexive) binary relation $<$ is called a partial order. Preferences are examples of partial orders. The order topology associated with a partial order $<$ (denoted by $\tau_{\text {or }}(<)$, or simply $\tau_{\text {or }}$ if no confusion is possible) is the topology which has the family formed by $X$ and all the subsets $\{a \in X: a<x\}$ and $\{a \in X: x<a\}$ with $x$ ranging over $X$ as a subbase of open sets. If $<$ is a linear order on $X$, we say that $\left(X,<, \tau_{\text {or }}(<)\right)$ is a LOTS, which stands for linearly ordered topological space.

For a partially ordered space $(X,<)$, we adopt the convention: given $A \subseteq X$ and $x \in X, x<A(A<x)$ if and only if $x<a(a<x)$ for all $a \in A$. Consequently, $x<\varnothing$ (and $\varnothing<x$ ). We let $A^{l}=\{x \in X: x<A\}, A^{u}=\{x \in X: A<x\}$. Consequently, $\varnothing^{l}=\varnothing^{u}=X$. The base $\mathscr{B}$ of $\tau_{\text {or }}$ formed by finite intersections of elements from the defining subbase is

$$
\mathscr{B}=\{\{x \in X: S<x<T\} \text { with } S, T \subseteq X \text { finite }\}=\left\{S^{u} \cup T^{l} \text { with } S, T \subseteq X \text { finite }\right\}
$$

Notice that $T=\varnothing$ and $S=\varnothing$ account for the cases when all the elements intersected are of the type $\{x \in X: a<x\}$ or the type $\{x \in X: x<b\}$. 
When we refer to the order topology derived from a preference, the use of the symbols $+\infty$ and $-\infty$ are defined by: each $x \in X$ satisfies $x<+\infty$ and $-\infty<x$. The base $\mathscr{B}$ of $\tau_{\text {or }}$ is expressed simply by $\mathscr{B}=\{\{x \in X: a<x<b\}$ with $a, b \in X \cup\{ \pm \infty\}\}$.

DEFINITION 3.1. A GPO-space (for Generalized Preference-Ordered space) is a triple $(X,<, \tau)$, where $<$ is a preference on $X$ and $\tau$ is a topology on $X$ such that $\tau_{\text {or }} \leq \tau$ and $\tau$ has a base formed by convex sets ( $C \subseteq X$ is convex if $p \preceq z \preceq q$ and $p, q \in C$ implies $z \in C$ ). A POTS (Preference-Ordered Topological Space) is a triple $\left(X,<, \tau_{\text {or }}\right)$, where $<$ is a preference on $X$.

If $<$ is a linear order, we obtain a GO-space and a LOTS, respectively (as defined e.g., in Faber [7]).

REMARK. Notice that, in any GPO-space, $\sim$ saturates the topology. Indeed, if $y \in A$, $A \in \tau$ and $x \sim y$, then there exists $C$ convex in $A$ such that $y \in C$. Now, $y \preceq x \preceq y$ implies $x \in C \subseteq A$.

A construction inspired in Faber [7, Prop. 2.1.2] permits one to show that every GPOspace can be topologically embedded in a POTS, i.e., in a set endowed with an order topology derived from a preference (and, conversely, every subspace of a GPO-space is clearly a GPO-space). Besides, we obtain a further characterization in terms of bases:

Proposition 3.1. Let $X$ be a set ordered by a preference < and $\tau$ a topology on $X$. Then $(X,<, \tau)$ is a GPO-space if and only if $\tau$ has a base consisting of all the subsets $\{x \in X: a<x<b\}$ with $a, b \in X \cup\{ \pm \infty\}$ and possibly some subsets of any of the following three types:

$$
\begin{aligned}
& \{x \in X: a<x \preceq b\} \text { with } a \in X \cup\{-\infty\} \text { and } b \in X \\
& \{x \in X: a \preceq x<b\} \text { with } b \in X \cup\{+\infty\} \text { and } a \in X \\
& \{x \in X: a \preceq x \preceq b\} \text { with } a, b \in X \text {. }
\end{aligned}
$$

Proof. From (P.1) above, all those sets are clearly convex. The fact that all the subsets of the type $\{x \in X: a<x<b\}$ are open is equivalent to $\tau_{\mathrm{or}} \leq \tau$. These two arguments justify the sufficiency of the claimed condition.

For the necessary condition, it suffices to show that the set $\mathscr{B}$ of all the open subsets that have any of those forms is a base of $\tau$. To this end, let $A \in \tau$ and $y \in A$. We find some $B \in \mathscr{B}$ with $y \in B \subseteq A$. There exists $C$ open and convex such that $y \in C \subseteq A$, and four cases may happen:

(i) There are $c, c^{\prime} \in C$ with $c<y<c^{\prime}$. Then $y \in B:=\left\{x \in X: c<x<c^{\prime}\right\} \subseteq C \subseteq A$ by the convexity of $C$. Now, $B \in \mathscr{B}$ since $B \in \tau_{\text {or }} \leq \tau$ and it has the required form.

(ii) There is $c \in C$ with $c<y$ and there is no $c^{\prime} \in C$ with $y<c^{\prime}$. Then $B=\{x \in$ $X: c<x \preceq y\}$ satisfies $B=C \cap\{x \in X: c<x\}$, by the convexity of $C$. Thus, $B$ is the intersection of two open sets and, therefore, as it fell within one of the required forms, $B \in \mathscr{B}$. Furthermore, $y \in B \subseteq C \subseteq A$.

(iii) There is $c^{\prime} \in C$ with $y<c^{\prime}$ and there is no $c \in C$ with $c<y$. The argument is analogous to that of case (ii).

(iv) There is no $c^{\prime} \in C$ with $y<c^{\prime}$ and there is no $c \in C$ with $c<y$. Then, as 
$C$ is convex, $C=\{x \in X: x \sim y\} \in \tau$ and $C \in \mathscr{B}$ (since $C=\{x \in X: y \preceq x \preceq y\}$. Furthermore, $y \in C \subseteq A$.

The strongest topology for which $(X,<, \tau)$ is a GPO-space is the indifference topology, which is defined by $\tau_{\sim}:=$ \{unions of equivalence classes by $\sim$. The coarsest one is obviously $\tau_{\text {or }}(<)$ itself. Corollary 4.1 exhibits two cases in which the topology $\tau$ of a GPO-space must equal $\tau_{\text {or }}(<)$, namely, when $\tau$ is connected and when $\tau$ is compact.

Recall that a map $f:(X,<) \longrightarrow\left(X^{\prime},<^{\prime}\right)$ between partially ordered sets is orderpreserving if $x<y$ implies $f(x)<^{\prime} f(y)$. If, furthermore, $f(x)<^{\prime} f(y)$ implies $x<$ $y$, we say that $f$ is an order-equimorphism.

Proposition 3.2. Let $f:(X,<) \longrightarrow\left(X^{\prime},<^{\prime}\right)$ be an epijective, order-preserving map between sets ordered by preferences. Then the following statements are equivalent:

(i) $f$ is an order-equimorphism

(ii) $f$ is a saturated identification with $\tau_{\text {or }}(<), \tau_{\text {or }}\left(<^{\prime}\right)$.

Proof. (i) $\Rightarrow$ (ii) We take $\mathscr{B}=\{\{x \in X: S<x<T\}$ with $S, T \subseteq X$ finite $\}$ as a base of $\tau_{\text {or }}(<)$ and $\mathscr{B}^{\prime}=\left\{\left\{x^{\prime} \in X^{\prime}: S^{\prime}<^{\prime} x^{\prime}<^{\prime} T^{\prime}\right\}\right.$ with $S^{\prime}, T^{\prime} \subseteq X^{\prime}$ finite $\}$ base of $\tau_{\text {or }}\left(<^{\prime}\right)$.

Now, $f$ is continuous: given $B^{\prime} \in \mathscr{B}^{\prime}$, we can write $B^{\prime}=\left\{x^{\prime} \in X^{\prime}: S^{\prime}<^{\prime} x^{\prime}<^{\prime} T^{\prime}\right\}$ with $S^{\prime}, T^{\prime} \subseteq X^{\prime}$ finite. There are $S, T \subseteq X$ finite such that $f(S)=S^{\prime}$ and $f(T)=T^{\prime}$. Therefore, $f^{-1}\left(\left\{x^{\prime} \in X^{\prime}: S^{\prime}<^{\prime} x^{\prime}<^{\prime} T^{\prime}\right\}\right) \subseteq\{x \in X: S<x<T\}$ (since if $x \in f^{-1}\left(x^{\prime}\right)$ for some $S^{\prime}<^{\prime} x<^{\prime} T^{\prime}$, then $S^{\prime}<^{\prime} f(x)=x^{\prime}<^{\prime} T^{\prime}$, and, therefore, $S<x<T$ because $f$ is order-equimorphism) and the opposite inclusion is straightforward. Hence, it follows that $f^{-1}\left(B^{\prime}\right) \in \tau_{\text {or }}(<)$.

Moreover, $f$ is open: for every $S, T \subseteq X$, we have

$$
\left\{x^{\prime} \in X^{\prime}: f(S)<^{\prime} x^{\prime}<^{\prime} f(T)\right\} \subseteq f(\{x \in X: S<x<T\})
$$

(if $f(S)<^{\prime} x^{\prime}<^{\prime} f(T)$, then there exists $x \in X$ with $f(S)<^{\prime} x^{\prime}=f(x)<^{\prime} f(T)$, and, hence, $S<x<T)$. Thus, $f(\{x \in X: S<x<T\})=\left\{x^{\prime} \in X^{\prime}: f(S)<^{\prime} x^{\prime}<^{\prime} f(T)\right\} \in$ $\tau_{\text {or }}\left(<^{\prime}\right)$ follows immediately. Therefore, $f(B) \in \mathscr{B}^{\prime}$ if $B \in \mathscr{B}$.

Finally, $A=f^{-1} f(A)$ for all $A \in \tau_{\text {or }}(<)$ : if $x \in f^{-1} f(A)$, then $f(x)=f(y)$ for some $y \in A$, and there exist $S, T \subseteq X$ finite such that $y \in\{z \in X: S<z<T\} \subseteq A$. Reasoning as above, $S<x<T$ and $x \in\{z \in X: S<z<T\} \subseteq A$.

Now, (ii) $\Rightarrow$ (i): if there exist $x, y$ such that $x<y$ is false but $f(x)<^{\prime} f(y)$, then $x \sim y$ and $f^{-1}\left(\left\{x^{\prime}<^{\prime} f(y)\right\}\right) \notin \tau_{\text {or }}(<)$ (because $y \sim x$ and $x \in f^{-1}\left(\left\{x^{\prime}<f f(y)\right\}\right)$ open imply that since $\sim$ saturates $\left.\tau_{\text {or }}(<), y \in f^{-1}\left(\left\{x^{\prime}<^{\prime} f(y)\right\}\right)\right)$; but this contradicts the continuity of $f$ because $\left\{x^{\prime}<^{\prime} f(y)\right\} \in \tau_{\text {or }}\left(<^{\prime}\right)$.

Notice that the implication (i) $\Rightarrow$ (ii) holds for any partially ordered set, as proved in Alcantud and Gutiérrez [2, Prop. 2]. Further conditions equivalent to (i) and (ii) are provided by [2, Prop. 3].

In applying Theorem 2.1 to the study of GPO-spaces, our main tool is the next Corollary. Given a preference $<$ on $X$, a linear order $<^{\prime}$ is naturally induced on $X / \sim$ (cf. Fishburn [8]). Thus:

COROLLARY 3.1. If $<$ is a preference on $X$ and $<^{\prime}$ is the induced linear order on $X / \sim$, 
then the projection map $p: X \rightarrow X / \sim$ is a saturated identification with the respective order topologies.

4. Consequences for GPO-spaces. Now, we are prepared to deduce the announced topological properties of GPO-spaces.

LEMMA 4.1. Let $(X,<, \tau)$ be a GPO-space. Then $\tau$ is $T_{0}$ if and only if $<$ is a linear order.

Proof. It is well known (cf. Birkhöff [3, Ch. X, Thm. 11]) that if the order is linear, then $\tau$ is $T_{0}$. Moreover, if $\tau \sim$ is $T_{0}$ then $<$ is obviously a linear order. As we showed in the comment following Proposition 3.1 that $\tau \leq \tau_{\sim}$, the necessity of the condition follows immediately.

Proposition 4.1. Let $\tau$ be a topology on $X,<$ a preference on $X$ and $<^{\prime}$ the linear order naturally induced on $X / \sim$. Then:

(i) $(X,<, \tau)$ is a GPO-space if and only if $\left(X / \sim,<^{\prime}, \tau_{\text {quot }}\right)$ is a GO-space. Moreover, in this case, the quotient map is a saturated identification.

(ii) $\tau=\tau_{\text {or }}$ if and only if $\tau_{\text {quot }}=\tau_{\text {or }}\left(<^{\prime}\right)$.

Proof. (i) We denote by $p$ the projection onto the quotient set. We already observed that, in any GPO-space, the indifference relation saturates the topology, which means that $p$ is a saturated identification with $\tau, \tau_{\text {quot }}$.

Now, suppose that $(X,<, \tau)$ is a GPO-space. Let $\mathscr{R}$ be a base of $\tau$ formed by convex sets. We shall see that $\mathscr{B}^{\prime}=\{p(B): B \in \mathscr{B}\}$ is a base of $\tau_{\text {quot }}$ formed by convex sets. We only need to show that $p(B)$ is convex for every $B \in \mathscr{B}$, i.e., that if $a^{\prime} \preceq^{\prime} x^{\prime} \preceq^{\prime} b^{\prime}$ for some $a^{\prime}, b^{\prime} \in p(B)$, then $x^{\prime} \in p(B)$. Indeed, since there are $a, b \in B$ and $x \in X$ with $a^{\prime}=p(a), b^{\prime}=p(B), x^{\prime}=p(x)$ and, thus, with $a \preceq x \preceq b$, we have $x \in B$ (by convexity) and $x^{\prime} \in p(B)$. Hence, $\mathscr{B}$ is formed by convex sets. Now, in order to prove that $\left(X / \sim,<^{\prime}, \tau_{\text {quot }}\right)$ is a GO-space, we verify that $\tau_{\text {or }}\left(<^{\prime}\right) \leq \tau_{\text {quot }}$. From Corollary 3.1, $p$ is a saturated identification with the order topologies. Thus, for every $A \in \tau_{\text {or }}\left(<^{\prime}\right)$, we have $p^{-1}(A) \in \tau_{\text {or }}(<) \leq \tau$ and, therefore, $A=p\left(p^{-1}(A)\right) \in \tau_{\text {quot }}$ (since $p$ is open with $\tau$ and $\tau_{\text {quot }}$ ).

Conversely, suppose that $\left(X / \sim,<^{\prime}, \tau_{\text {quot }}\right)$ is a GO-space. Let $\mathscr{B}^{\prime}$ be a base of $\tau_{\text {quot }}$ formed by convex sets. We shall see that $\mathscr{B}=\left\{p^{-1}\left(B^{\prime}\right): B^{\prime} \in \mathscr{B}^{\prime}\right\}$ is a base of $\tau$ formed by convex sets. We only need to show that $p^{-1}\left(B^{\prime}\right)$ is convex for every $B^{\prime} \in \mathscr{B}^{\prime}$, i.e., that if $a \preceq x \preceq b$ for some $a, b \in p^{-1}\left(B^{\prime}\right)$, then $x \in p^{-1}\left(B^{\prime}\right)$. Indeed, as $p(a) \preceq^{\prime} p(x) \preceq^{\prime}$ $p(b)$ and $B^{\prime}$ is convex, it follows that $p(x) \in B^{\prime}$. Thus, $x \in p^{-1}\left(B^{\prime}\right)$. Hence, $\mathscr{B}$ is formed by convex sets. Now, in order to prove that $(X,<, \tau)$ is a GPO-space, we verify that $\tau_{\text {or }}(<) \leq \tau$. Since $\left(X / \sim,<^{\prime}, \tau_{\text {quot }}\right)$ is a GO-space, it follows that $\tau_{\text {or }}\left(<^{\prime}\right) \leq \tau_{\text {quot }}$. As $p$ is a saturated identification with the order topologies, for all $A \in \tau_{\text {or }}(<)$, we have $p(A) \in \tau_{\text {or }}\left(<^{\prime}\right) \leq \tau_{\text {quot }}$. Thus, $A=p^{-1}(p(A)) \in \tau$ (since $p$ is continuous with $\tau$ and $\tau_{\text {quot }}$ ).

(ii) Assume that $\boldsymbol{\tau}=\tau_{\text {or }}(<)$. Now, $p^{-1}(A) \in \tau=\tau_{\text {or }}(<)$ for every $A \in \tau_{\text {quot }}$ and then $A=p\left(p^{-1}(A)\right) \in \tau_{\text {or }}\left(<^{\prime}\right)$. Therefore, $\tau_{\text {or }}\left(<^{\prime}\right)=\tau_{\text {quot }}$. The converse implication follows from an analogous argument.

COROLLARY 4.1. If $(X,<, \tau)$ is a GPO-space and $\tau$ is either compact or connected, 
then $\tau=\tau_{\text {or }}(<)$.

Proof. Recall that if $\left(X,<^{*}, \tau^{*}\right)$ is a GO-space and $\tau^{*}$ is either compact or connected, then $\tau^{*}=\tau_{\text {or }}\left(<^{*}\right)$ (cf. Lutzer [14, Lem. 6.1]). The result, now, follows from Proposition 4.1.

COROLLARY 4.2. Let $(X,<, \tau)$ be a GPO-space. Then:

(i) If $\tau$ is connected then it is locally connected.

(ii) If $\mathrm{T}$ is separable then it is first countable.

Proof. (i) From (i) and (ii) in Proposition 4.1 and Corollary 4.1: $\tau=\tau_{\text {or }}(<)$ is connected implies that $\tau_{\text {or }}\left(<^{\prime}\right)$ is connected, and, therefore, (cf. Eilenberg [5, (8.1)]) the later is locally connected. Now, Theorem 2.1(iv) implies that $\tau$ is locally connected.

(ii) From (i) in Proposition $4.1,\left(X / \sim,<^{\prime}, \tau_{\text {quot }}\right)$ is a separable GO-space, and, therefore, (cf. Faber [7, Cor. to Prop. 2.2.3]) it is first countable. Now, Theorem 2.1(i) implies that $\tau$ is first countable.

The proof of Theorem 4.1 (that we shall introduce later) requires checking that GPOspaces satisfy property $\mathrm{MN}^{\prime}$. This follows from Proposition 4.2:

Proposition 4.2. If $f:(X, \tau) \longrightarrow\left(X^{\prime}, \tau^{\prime}\right)$ is a saturated identification and $X^{\prime}$ satisfies $\mathrm{MN}^{\prime}$, then $X$ satisfies $\mathrm{MN}^{\prime}$.

Proof. Given an assignment fulfilling property $\mathrm{MN}^{\prime}$ for $X^{\prime}$, and denoting by $W_{t}$ the open set corresponding to $t \in W \in \tau^{\prime}$, we define: for every $x$ and $U$ such that $x \in U \in \tau, U^{x}:=f^{-1}\left(f(U)_{f(x)}\right) \in \tau$. Then, if there exists $z \in U^{x} \cap V^{y}$, clearly $f(z) \in$ $\left(f(U)_{f(x)}\right) \cap\left(f(V)_{f(y)}\right)$; therefore, by $\mathrm{MN}^{\prime}$, either $f(x) \in f(V)$ and $x \in f^{-1} f(V)=V$, or $f(y) \in f(U)$ and $y \in f^{-1} f(U)=U$. Now, $X$ satisfies $\mathrm{MN}^{\prime}$ through the operator $M$ defined by $M(x, U):=U^{x}$ for all pairs $(x, U)$ with $x \in U \in \tau$.

Recall that a topological space is collectionwise normal if, for each discrete collection $\mathscr{H}$ of closed subsets of $X$, there is a collection $\mathscr{D}=\{D(H): H \in \mathscr{H}\}$ of pairwise disjoint, open subsets of $X$ with the property that $H \subseteq D(H)$ for every $H \in \mathscr{H}$. Under these conditions, $\mathscr{H}$ is discrete if each point of $X$ has a neighborhood that meets at most a finite number of members of the collection.

THEOREM 4.1. Every GPO-space satisfies $\mathrm{MN}^{\prime}$, and, thus, it is monotonically normal, (hereditarily) normal, (completely) regular and (hereditarily) collectionwise normal.

Proof. From Heath et al. [10, Cor. 5.6] (due to Heath and Lutzer), every GO-space is monotonically normal. Because GO-spaces are $T_{1}$, they also satisfy $\mathrm{MN}^{\prime}$. Propositions 4.1 and 4.2 imply that GPO-spaces satisfy $\mathrm{MN}^{\prime}$ and, therefore, they are monotonically normal. The proof of Heath et al. [10, Thm. 3.1] (due to Zenor) yields the last statement, because it shows that monotone normality MN implies hereditary collectionwise normality without requiring the $T_{1}$ axiom.

Since every GO-space is ( $T_{1}$ and) hereditarily normal, Theorem 2.1 implies that GPOspaces are hereditarily normal and (completely) regular.

REMARK. If $(X,<, \tau)$ is a GPO-space, we have shown in Proposition 4.1 that $(X / \sim$, $\left.<^{\prime}, \tau_{\text {quot }}\right)$ is a GO-space and the projection $p:(X, \tau) \rightarrow\left(X / \sim, \tau_{\text {quot }}\right)$ is a saturated 
identification. Therefore, Theorem 2.1 implies that $\tau$ is first (second) countable, separable, (countably, locally) compact, Lindelöff or (locally) connected if and only if $\boldsymbol{\tau}_{\text {quot }}$ is the same. Besides, the same can be said of $\tau_{\text {or }}(<)$ and $\tau_{\text {or }}\left(<^{\prime}\right)$ by repeating the argument and using Corollary 3.1.

Therefore, in order to study the properties of GPO-spaces (including POTS), we can benefit from the corresponding results for GO-spaces and LOTS. Some other relevant results in this latter setting include Birkhöff's characterization of compactness and connectedness for LOTS [3, Thm. 12 X.7, Thm. 14 X.7], Faber's characterizations of compactness and connectedness for GO-spaces [7, Thm. 2.4.1, Thm. 2.4.2], and van Wouwe's characterization of countable compactness for GO-spaces [21, Thm. 2.2.1].

ACKNOWLEDGEMENT. A previous version of this work has appeared in the CDAM (Center for Discrete and Applied Mathematics) Research Report Series, The London School of Economics (n. 96, November 1995). Thanks are due to J. M. Gutiérrez for the considerable help he has extended. This research was supported by DGICYT Project PB94-1385.

\section{REFERENCES}

[1] J. C. R. Alcantud, Topological separability and axioms of countability in GPO-spaces, Bull. Austral. Math. Soc. 55 (1997), no. 1, 131-142. CMP 97 06. Zbl 970.50578.

[2] J. C. R. Alcantud and J. M. Gutiérrez, Saturated identifications and topological properties of GPO-spaces, november 1995, CDAM Research Report no.96 The London School of Economics.

[3] G. Birkhöff, Lattice Theory, American Mathematical Society, New York, 1940. MR 1,325f. Zbl 063.00402.

[4] R. Duda, On ordered topological spaces, Fund. Math. 63 (1968), 295-309. MR 38\#3833. Zbl 177.50801 .

[5] S. Eilenberg, Ordered topological spaces, Amer. J. Math. 63 (1941), 39-45. MR 2,179e. Zbl 024.19203.

[6] M. Erné, Separation axioms for interval topologies, Proc. Amer. Math. Soc. 79 (1980), no. 2, 185-190. MR 81d:54012. Zbl 431.54016.

[7] M. J. Faber, Metrizability in generalized ordered spaces, Mathematical Centre Tracts, no. 53, Mathematisch Centrum, Amsterdam, 1974. MR 54 6097. Zbl 282.54017.

[8] P. C. Fishburn, Utility theory for decision making, Publications in Operations Research, no. 18, John Wiley \& Sons, Inc., New York, 1970. MR 41\#9401. Zbl 213.46202.

[9] G. Gruenhage, Generalized metric spaces, Handbook of set theoretic topology (Amsterdam), Generalized metric spaces, North Holland, 1984, pp. 423-501. MR 86h:54038. Zbl 555.54015.

[10] R. W. Heath, D. J. Lutzer, and P. L. Zenor, Monotonically normal spaces, Trans. Amer. Math. Soc. 178 (1973), 481-493. MR 51 9030. Zbl 269.54009.

[11] G. Herden, On the existence of utility functions. II, Math. Social Sci. 18 (1989), no. 2, 107117. MR 91c:90012. Zbl 692.90006.

[12] M. Kolibiar, Intrinsic topologies on ordered sets, Acta Math. Univ. Comenian. 39 (1980), 151-157. MR 82h:54051. Zbl 515.54002.

[13] L. A. Steen and J. A. Seebach, Jr., Counterexamples in topology, second ed., SpringerVerlag, New York, 1978. MR 80a:54001. Zbl 386.54001.

[14] D. J. Lutzer, On generalized ordered spaces, Dissertationes Math. Rozprawy Mat. 89 (1971), 1-36. MR 48 3018. Zbl 228.54026.

[15] I. L. Lynn, Linearly orderable spaces, Proc. Amer. Math. Soc. 13 (1962), 454-456. MR 25\#1536. Zbl 108.35505. 
[16] G. Mehta, Ordered topological spaces and the theorems of Debreu and Peleg, Indian J. Pure Appl. Math. 14 (1983), no. 9, 1174-1182. MR 84i:54035. Zbl 515.54024.

[17] L. Nachbin, Topology and order, Robert E. Krieger Publishing Co. Inc., New York, 1976, Translated from the Portuguese by Lulu Bechtolsheim. Reprint of the 1965 edition. MR 54 3667. Zbl 333.54002.

[18] E. S. Northam, The interval topology of a lattice, Proc. Amer. Math. Soc. 4 (1953), 824-827. MR 15,244a. Zbl 051.26002.

[19] O. Frink, Jr., Topology in lattices, Trans. Amer. Math. Soc. 51 (1942), 569-582. MR 3,313b. Zbl 061.39305.

[20] J. van Dalen and E. Wattel, A topological characterization of ordered spaces, General Topology and Appl. 3 (1973), 347-354. MR 49 6182. Zbl 272.54026.

[21] J. M. van Wouwe, GO-spaces and generalizations of metrizability, Math. Centre Tracts, vol. 104, Mathematisch Centrum, Amsterdam, 1979. MR 80m:54046. Zbl 438.54030.

[22] A. Wilansky, Topology for analysis, Robert E. Krieger Publishing Co., Melbourne, 1983. MR 85d:54001. Zbl 512.54001.

[23] S. Willard, General topology, Addison Wesley Publishing Co., Mass. London Don Mills, 1970. MR 41\#9173. Zbl 205.26601.

ALCANTUD: FACULTAD DE ECONOMIA Y EMPRESA, UNIVERSIDAD DE SALAMANCA, E 37008 SALAMANCA, SPAIN

E-mail address: jcr@gugu.usa1.es 


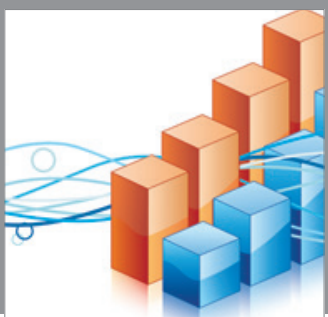

Advances in

Operations Research

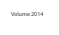

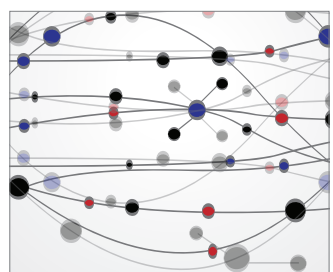

\section{The Scientific} World Journal
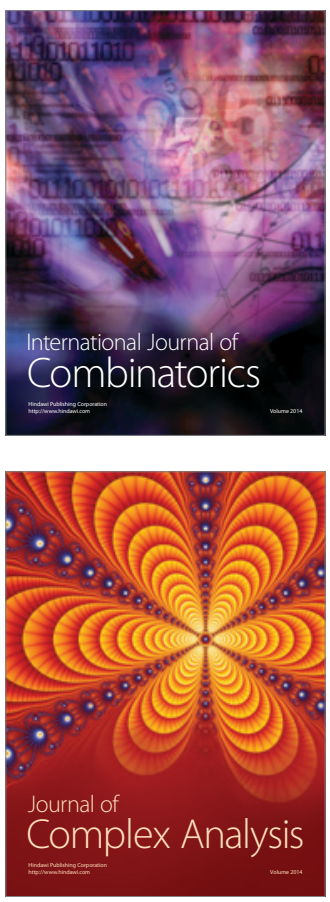

International Journal of

Mathematics and

Mathematical

Sciences
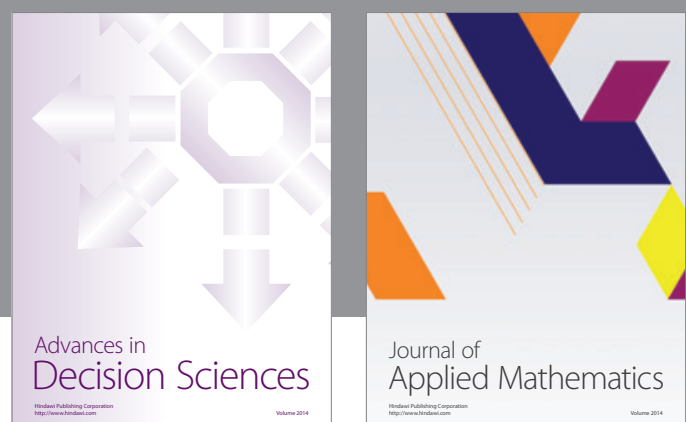

Journal of

Applied Mathematics
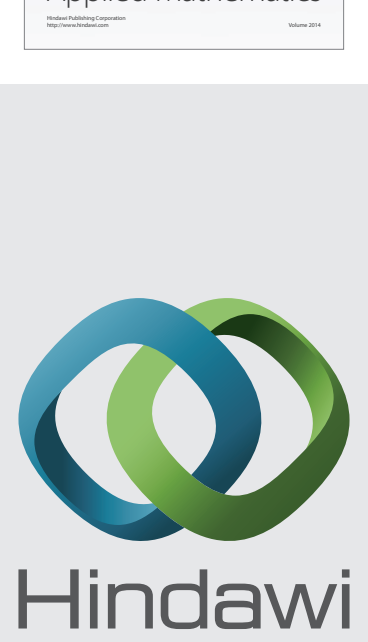

Submit your manuscripts at http://www.hindawi.com
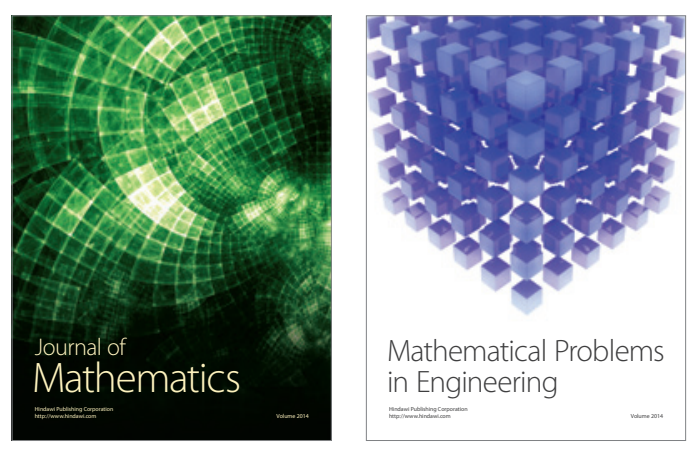

Mathematical Problems in Engineering
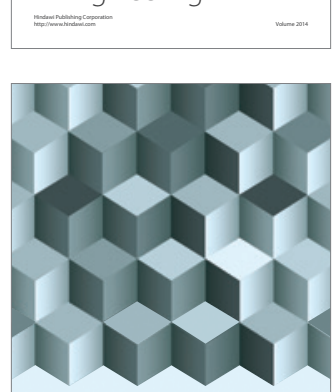

Journal of

Function Spaces
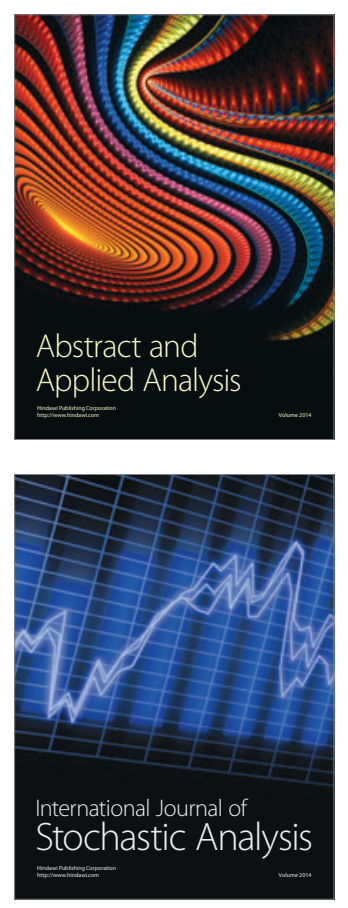

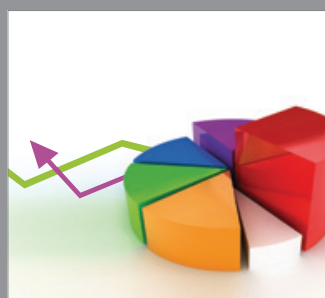

ournal of

Probability and Statistics

Promensencen
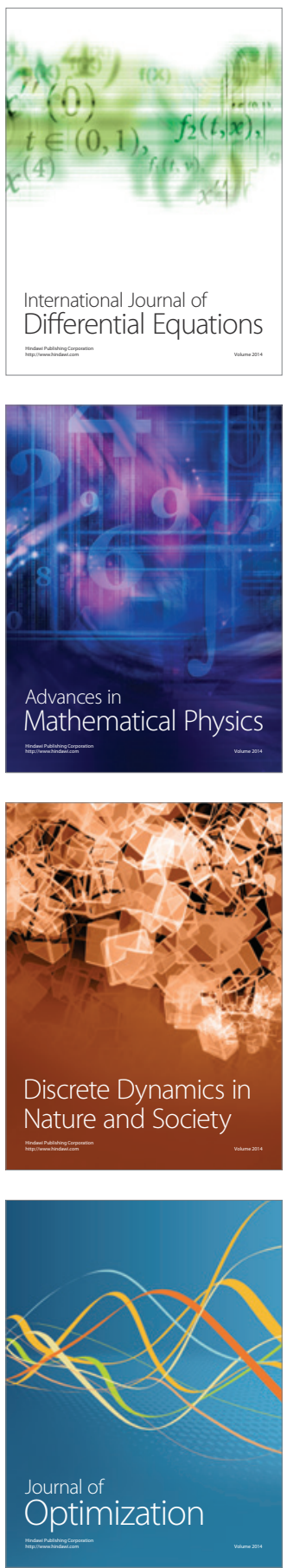